Basrah Journal Of Surgery
I read for you

Bas J Surg, June, 24, 2018

\title{
MANAGEMENT OF CRISES DURING ANESTHESIA AND SURGERY. PART XIV: ALLERGY \& ANAPHYLAXIS
}

\author{
Salam N Asfar@ \& Jasim M Salman ${ }^{\#}$ \\ ${ }^{\circledR} \mathrm{MB}, \mathrm{ChB}, \mathrm{MSc}$, Professor of Anesthesiology, College of Medicine, University of Basrah. ${ }^{*} \mathrm{MB}, \mathrm{ChB}$, \\ DA, FICMS, Assist. Prof. \& Consultant Anesthesiologist, College of Medicine, University of Basrah, \\ Basrah, IRAQ.
}

\begin{abstract}
A lhough serious allergic reactions to drugs and materials used in anesthesia and Asurgery are rare but it could be life threatening. Anesthesiology personnel should be trained for immediate recognition and successful management of this situation. Careful history taking in atopic patients is vital. Investigations should be done later to find out the causative agent to avoid or treat such condition.

Causes: Agents concerned in anaphylactic reactions during anesthesia ${ }^{1,2}$ :

*Neuromuscular blocking agents: mainly Succinylcholine, Rocuronium, Atracurium, Vecuronium, Pancuronium, Mivacurium, and Cisatracurium.

*Antibiotics: Including; Penicillin, Cephalosporin, Quinolone, Vancomycin, and Rifamycin.

*Hypnotics: Propofol, Thiopental and Midazolam

*Opioids : Fentanyl, Sufentanil, and Morphine

*Blood products transfusion

*Colloids : Gelatin and Hetastarch plasma volume expanders

*Latex (natural rubber)

* Other agents such as: Bupivacaine, Protamine, Hyaluronidase and Methylene blue
\end{abstract}

Signs: signs are graded according to the severity of allergic reaction ${ }^{3}$ :

\begin{tabular}{|c|c|c|c|}
\hline Grade 1 & Grade 2 & Grade 3 & Grade 4 \\
\hline $\begin{array}{l}\text { Cutaneous } \\
\text { - Erythema } \\
\text { - Urticaria } \\
\text { - Angioedema } \\
\text { face and lips }\end{array}$ & $\begin{array}{l}\text { Grade } 1 \text { signs } \\
\text { plus: } \\
\text { Cardiovascular } \\
\text { - Hypotension } \\
\text { - Tachycardia } \\
\text { Respiratory } \\
\text { - Wheezing. } \\
\text { - Pulmonary } \\
\text { edema }\end{array}$ & $\begin{array}{l}\text { Grade } 2 \text { signs } \\
\text { plus: } \\
\text { Cardiovascular } \\
\text { - Cardiovascular collapse } \\
\text { - Profound hypotension } \\
\text { - Bradycardia } \\
\text { - Dysrhythmia } \\
\text { Respiratory } \\
\text { - Bronchospasm; } \\
\text { - Hypoxia (SaO2 <92\%) } \\
\text { Neurologic } \\
\text { - Unconscious in recovery }\end{array}$ & $\begin{array}{l}\text { Cardiovascular } \\
\text { - Pulseless electrical } \\
\text { activity } \\
\text { - Cardiac arrest }\end{array}$ \\
\hline
\end{tabular}




\section{Management ${ }^{4,5}$ :}

\section{Primary treatment}

- Stop administration of substance

- Call for help, inform surgeon

- Trendelenberg position

- Airway management - oxygen

- Adrenalin: Give adrenaline bolus IV $0.001 \mathrm{mg} / \mathrm{kg}$ (adult dose $1 \mathrm{ml}$ of 1:10,000). Start adrenaline infusion $0.00015 \mathrm{mg} / \mathrm{kg} / \mathrm{min}$ (adult dose $1 \mathrm{ml} / \mathrm{min}$ of $1 \mathrm{mg}$ in $100 \mathrm{ml}$ ) and increase as necessary. Titrate against heart rate and blood pressure.

- Fluid Therapy: Crystalloid 10-20 ml/kg.

\section{Secondary treatment}

-Antihistamine: $\mathrm{H} 1$ antagonists: promethazine $0.3-1 \mathrm{mg} / \mathrm{kg}$

$\mathrm{H} 2$ antagonists: ranitidine $0.5-1 \mathrm{mg} / \mathrm{kg}$

-Corticosteroids: Hydrocortisone up to $5 \mathrm{mg} / \mathrm{kg}$

-B2 agonists nebulisation: Salbutamol 5-10 $\mu \mathrm{g}$

\section{References}

1. Laxenaire MC, Mertes PM. Anaphylaxis during anaesthesia. Results of a two-year survey in France. British Journal of Anaesthesia, 2001;87:549-558.

2. Jenkins LC, Graves HB. Blood transfusion reactions during anaesthesia; a clinical study. Canadian Anaesthetists' Society Journal, 1961;8:492-500.

3. Dippenaar JM, Naidoo S. Allergic reactions and anaphylaxis during anaesthesia, Current Allergy \& Clinical Immunology, 2015;28:18-22.

4. Panjo GB, Crisafulli J, Calminiti L, Marseglia GL, Cardinale F, Paravati F, Caffarelli C. Peri-operative allergy: Therapy. Int J Immunopath Phar. 2011;24(3):101-104.

5.Currie M, Kerridge RK, Bacon AK. Crisis management during anaesthesia: anaphylaxis and allergy. Downloaded from qshc.bmj.com on May 20, 2010. Published by group.bmj.com. 